\title{
Fate, uptake, and distribution of nanoencapsulated pesticides in soil-earthworm systems and implications for environmental risk assessment
}

\begin{abstract}
Nanopesticides are novel plant protection products offering numerous benefits. Because nanoparticles behave differently from dissolved chemicals, the environmental risks of these materials could differ from conventional pesticides. We used soilearthworm systems to compare the fate and uptake of analytical-grade bifenthrin to that of bifenthrin in traditional and nanoencapsulated formulations. Apparent sorption coefficients for bifenthrin were up to 3.8 times lower in the nano treatments than in the non-nano treatments, whereas dissipation half-lives of the nano treatments were up to 2 times longer. Earthworms in the nano treatments accumulated approximately $50 \%$ more bifenthrin than those in the non-nano treatments. In the non-nano treatments, most of the accumulated material was found in the earthworm tissue, whereas in the nano treatments, the majority resided in the gut. Evaluation of toxicokinetic modeling approaches showed that models incorporating the release rate of bifenthrin from the nanocapsule and distribution within the earthworm provided the best estimations of uptake from the nano-formulations. Overall, our findings indicate that the risks of nanopesticides may be different from those of conventional formulations. The modeling presented provides a starting point for assessing risks of these materials but needs to be further developed to better consider the behavior of the nanoencapsulated pesticide within the gut system.
\end{abstract}

Keyword: Earthworms; Eisenia fetida; Lumbricus terrestris; Nanoencapsulation; Nanopesticides; Synthetic pyrethroids; Toxicokinetic modeling 